\title{
An Intelligent Alarm and Messaging Based Surveillance System for Fall Detection and Absence Recognition of Unaccompanied Child
}

\author{
Ali Javed, Rabeea Islam \\ Department of Software Engineering, University of Engineering \& Technology Taxila \\ Email: ali.javed@uettaxila.edu.pk; rabeeakhan22@gmail.com
}

\begin{abstract}
Video analytics refers to process the videos intelligently. Video analytics has its most important usage in the field of the surveillance. Surveillance has been used in various areas and one of them is the detection of unintentional fall of patients, senior citizens and children which can cause serious injuries and health threats to children as well as to old persons. Developed countries are progressing in the Surveillance and activity monitoring. But there are limitation and facing problems under certain circumstances. Advancement in the field of computer vision and the prominent decrease in the prices of digital cameras assisted and motivated researchers to propose very useful algorithms for fall detection. The proposed research work is based on the combination of motion history images and eclipse centroid calculation to detect the fall efficiently. The proposed system provides very effective and efficient results on the video sequences of simulated falls.
\end{abstract}

Index Terms - Absence Recognition, Fall Detection, Surveillance

\section{INTRODUCTION}

There has been a lot of research work done in Computer Vision and image processing field in the past few decades because of the availability and price decay in digital equipment. The proposed research work is our contribution towards knowledge in the area of tracking and activity recognition. The advancement in technology has motivated research scholars to provide solutions related to human tracking and activity recognition that were not considered feasible some decades ago. Surveillance systems have provided applications for human tracking, activity monitoring, fall detection and so on. It is one of the major advancement in the field of Computer Vision.

Human tracking and fall detection applications can be used in various areas like for surveillance purposes, for pedestrian movement analysis, for patients and elderly person's movements in the hospitals and homes, etc. These systems have been deployed in Military Organizations, in Airports, in educational institutions and corporate offices.
Due to the existence of many day care centers now a days there is a huge demand for systems which can monitor the activities of children in the day care to avoid any injury to children. The instant cure of wounded child is extremely perilous, that's why we have to identify the fall as soon as possible to minimize any threat of severe damage. Though it looks quite easy to perceive the fall detection but it is difficult to estimate it accurately because of the diverse portions of human body it requires efficient measurement approaches.

The proposed research work targets the area of for child monitoring, fall detection and absence recognition. This application has its significance in day care centers and even at homes for observing the child posture and behavior to detect fall of child and child absence from a particular area. It is very difficult to keep eye on every child in house especially in Child Care Centers where children are in large amount. The proposed research work provides an efficient and effective solution for child monitoring and activity recognition. The proposed system monitors the child and generates an Alarm in case the child falls or leaves the specified portion in the room.

\section{LITERATURE REVIEW}

The proposed research work covers the situations of unintentional fall detection which involves the work related to human detection, posture analysis and motion estimation. Many researchers offered their algorithms for the detection of the human motion. Different methods are used to detect the motion of a human like understanding human's activities and train the machine to detect the movement from the activities.

There are many systems based on observing humans and understanding their activities to function, they require methods for detecting people from a given input image or a video. Zhuo-LIN JIANG [1] describes an effective solution to correctly distinguish people from other moving objects in indoor environments, including shadows, sudden changes of light and movements. Dalal \& Triggs [2] presented a human detection algorithm with excellent results shown for detection. Basically a dense grid is used in this method which is based on the histograms of Oriented Gradients, computed over blocks 
of size $16 \times 16$ pixels to represent a detection window. Paul viola [3] presents a machine learning approach for visual object detection capable of processing images extremely rapidly and achieving high detection rates.

Child's posture analysis is another major phase in the proposed research work after child detection. Child Posture Analysis has been accomplished in the proposed system to achieve the task of detecting child fall.

Child's posture needs to be examined very keenly and have to consider all scenarios for a successful research because children can behave in a much unexpected way and to tackle that problem our system should work properly on every posture. Chen and Schonfeld proposed a framework for providing both video tracking and pose estimation [4]. Jamil and Adnan [5] proposed an approach to tackle the issue of unintentional falls of patients. They proposed a combined approach of motion gradients and human shape features variation which gives quite good results.

Child posture analysis involves detecting and analysis the features of the child that are important in falling of a child. It includes the posture of many body parts including legs, arms and back portions. In this era, computing based on the scientifically proved methods gains supreme importance in our life. These methods help us to do all the intelligent computing for human computer interaction. Hence, postures are ambiguous and incompletely specified. There have been varied approaches to handle gesture recognition like mathematical models based on hidden Markov chains [6].

To estimate the motion from the video, we have to work on the frames extracted from the video and most of the time those consecutive images almost contains the same objects as previous frame contains [7]. Estimation basically is a process which examines the motion of objects to predict the vector for estimated motion. There is another technique used in this process which is called motion compensation, it uses the motion knowledge of the object to achieve the data compression. The most obvious problem in this process is the redundancy found in the consecutive frames because mostly consecutive frames contain same information. Interframe coding can be used to overcome this issue. Interframe coding is a technique to remove the temporal redundancy found in the consecutive frames to accomplish higher compression. [8]

There are some methods used for the object detection and recognition. Support vector machine is one technique which can be used for object detection and recognition with better accuracy. SVM takes different classes of data as input, analyzes the data, process it and finally generates output. More precisely, SVM draws a plane or model in space and then this plane is basically used for the matching purpose. This will further used to classify the pattern in different groups. This is also used for the machine learning, SVM tells that the given set of data whether belongs to a certain class or not. This is less effective due to time consumption. SVM related work has been presented in $[9,10]$.

SIFT is another technique used for object detection. This method includes many features of the objects like its color, coordinates in space etc. basically it uses many characteristics of the image for object detection. Due to its outstandingly correct output it is used many applications like robotics, image stitching and video surveillance [11]. One of the significant drawback of SIFT is maintenance of feature set once defined its pretty difficult to change it because the results of detection gives many false alarms in case of modification in the feature set after definition.

Artificial Neural network detection basically uses the hypothetical pattern which is created in space and then to the object is matched for object recognition. It works just like the nervous system, where very complex small neurons are connected with each other and form a pattern. This method is complex one but its accuracy is outstanding [6]. This method is a different from the other algorithmic approaches because all the algorithms handle the problems for which they are designed. On the other hand artificial neural networks can handle the problems based on the examples provided to it. You just give it some examples and this method will solve the problem on the basis of these examples. That's why the selection of examples is very important and the time to find the solution based on the selection of the examples. The only drawback is the unpredictable nature of output results in this case.

\section{SYSTEM ARCHITECTURE}

The proposed research work involves the detection of Child falling and absence recognition from a particular area. The proposed algorithm comprises of different phases. The system architecture of the proposed algorithm is presented below in fig. 1. The proposed system captures the scene of the bedroom in image acquisition phase. The captured video undergoes scenario detection phase after image enhancement in the preprocessing stage. Different coordinates of the frame is calculated in this phase. These coordinates facilitated to identify the location of bed in the room. 


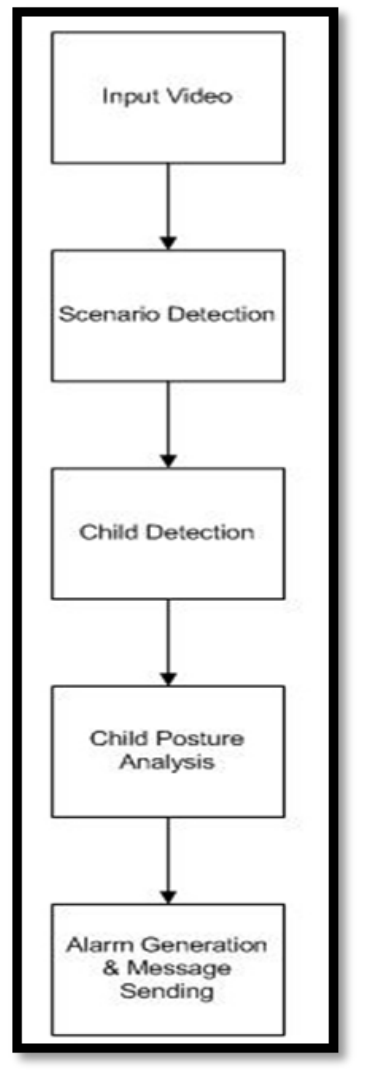

Figure 1. System Architecture

Child detection is accomplished in the next phase, followed by Child posture analysis. The Child Detection phase is explicated in fig. 2 .

The frames of the video have been analyzed and child detection has achieved successfully from the captured scene. If the Child is found in the captured scene then the frame undergoes motion history analysis. This motion history calculation determined that either child is moving towards the edge of bed or not. If the Child is going towards wall, an alarm message is generated to inform the care taker to perform necessary actions to prevent any injury to child.

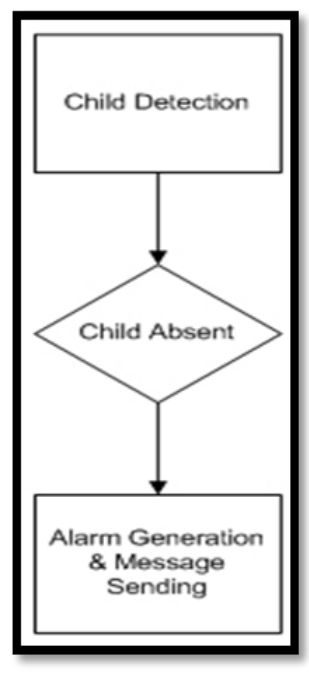

Figure 2 Child Detection
The next phase involves the posture analysis of child to decide whether the child is going to fall or not. If Child is detected in bend position and going to fall, an alarm will be generated and alert message will be send. If child is not detected in frame, the frame is discarded and next frame is selected for processing. The flow of child posture analysis for fall detection is shown in fig. 3.

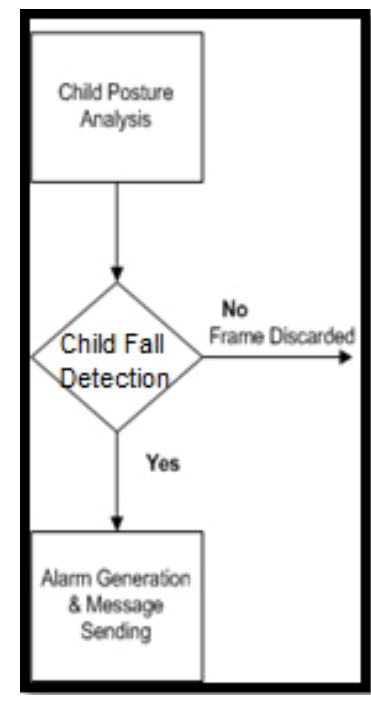

Figure 3. Child Posture Analysis

\section{PROPOSED METHODOLOGY}

\section{A. Image Acquisition}

The proposed system captures the scene by using static digital camera which captures video at a resolution of 640 x 480 pixels. The camera is fixed and capturing video at a frame rate of 25 frames per second. Since we want to develop economical system that's why we used static digital camera for scene capturing.

Human detection and tracking can become a very complex chore in the presence of the noise, low illumination and shadows. The proposed system achieves the desired objectives even in adverse conditions like noise, low illumination and shadows.

\section{B. Image Preprocessing}

The captured video is enhanced in the preprocessing stage to reduce the noise and to overcome any issues regarding low level illumination and reflection.

Gaussian Low pass filter of size $5 \times 5$ is applied for noise reduction in the preprocessing phase. The application of Gaussian filter on the input video enhances the video by achieving more smoothing and reduces the noise. This preprocessing phase assists to improve the quality of video resulting in better processing of the video for child detection and posture analysis.

\section{Child Detection}

The gathered frames of the video are processed first for removal of foreground portion, the area of interest, 
which is child in our case, and then Gaussian is generated. The boundary of the frame is detected for identifying the child.

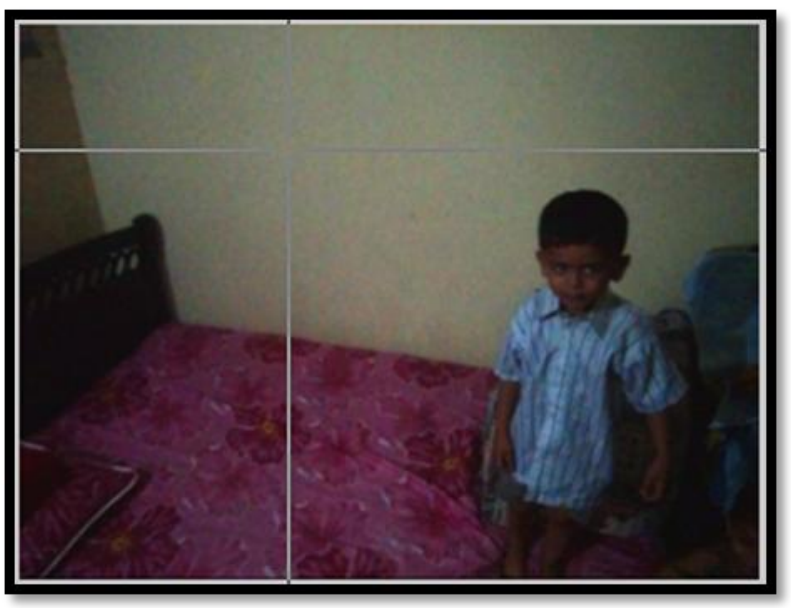

Figure 4. Boundary Selection

Fig. 4 shows the boundary selection of the frame processed to define the boundary line for the child.

\section{Child Posture Analysis}

The values gathered from the above results are processed further to generate the standard deviation of the values. The plot of the processed results representing the statistics is shown by the graph in fig. 5 .

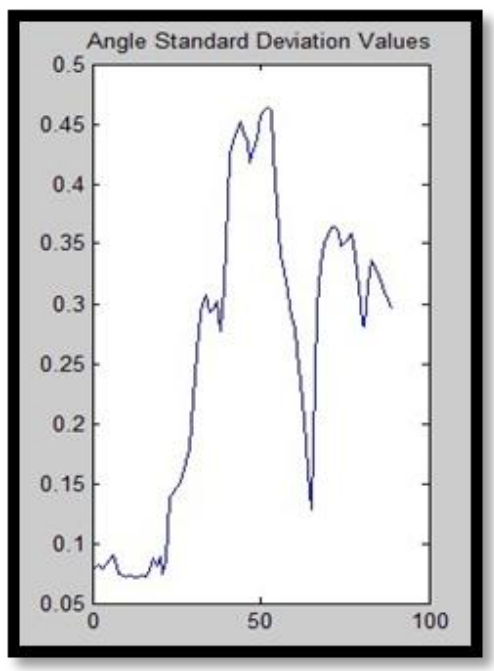

Figure. 5 Standard De viation Analysis

The standard deviation vectors for the orientation and eccentricity has been calculated and represented as the following parameters. Time $=1 \mathrm{~s}=25$ frames.

The results of Standard Deviation have been further analyzed to detect motion coefficient of the input video frame as shown in the fig. 6 .

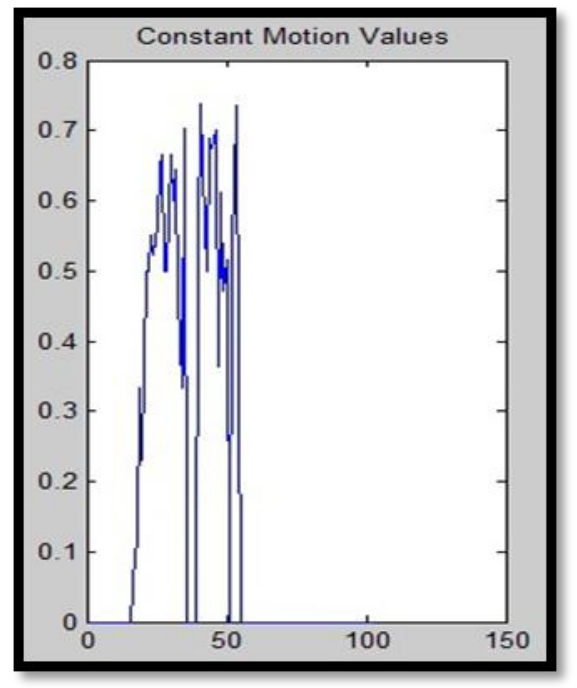

Figure 6. Constant Motion Analysis

The input video frame is processed for fall detection. The image shown in fig 7 below that the frame has successfully detected the child fall. This has been achieved by detecting the collision with the object.

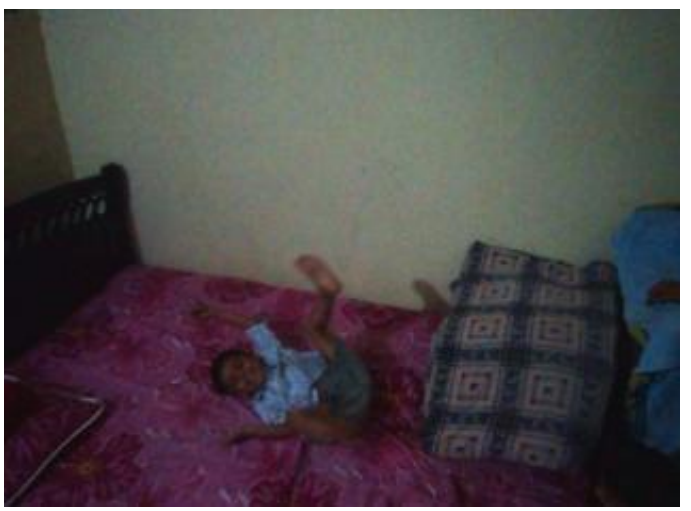

Figure 7. Child Fall Detection

The motion history of the image has been calculated once child fall event is detected. The output has been initialized, MHI a.k.a. $\mathrm{H}(\mathrm{x}, \mathrm{y}, \mathrm{t}, \mathrm{T})$. The initialization process is followed by parameters definition for further processing. The motion history image MHI parameter $\mathrm{T}$ representing the number of frames is being considered here. We have assumed maximal value of MHI. The dimensions of the video have been obtained, after the assumption of maximum value, by extracting the first frame from the video.

Compute $\mathrm{H}(\mathrm{x}, \mathrm{y}, 1, \mathrm{~T})$, the first MHI of video, start global loop for each frame, load current frame from image cell, begin looping through each point. The MHI of the resultant image is shown below in fig 8 . 


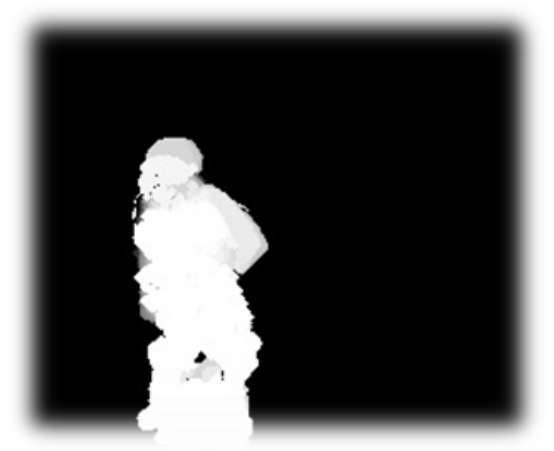

Figure 8. MHI of Child Detection

The collision detection of falling child has been calculated after MHI. To achieve this purpose following steps have been performed.

1. Define Number of Frames

2. Define the output, centroid array

3. Start global loop for each frame

4. Load current frame from image cell

5. Get dimensions of the frame

6. Definition of CENTROID $=$ SUM(mass of the point $*$ position of the point) / SUM(mass of the point)

7. Define the weighted mass, which is the product of each 'mass' weighted\% by its position

8. Define the sum of masses, a variable to be used in upcoming loop

9. In this case, the 'mass' of each point is either 1 or 0 , so the sum of the masses will effectively be the sum of all the 1's in the frame, or equivalently, the number of 1 's in the frame.

10. Begin looping through each point
i. X Dimension
ii. Y Dimension

11. Calculate Centroid in $\mathrm{x}$ and $\mathrm{y}$ dimensions

The results are shown in fig. 9.

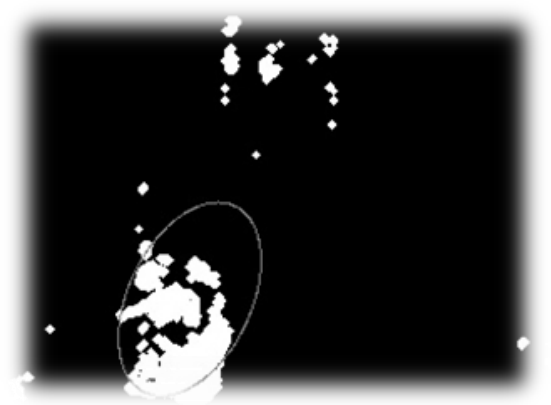

Figure 9. Child Collision Detection

The statistics of the proposed system have been presented and mentioned in the fig 10 .

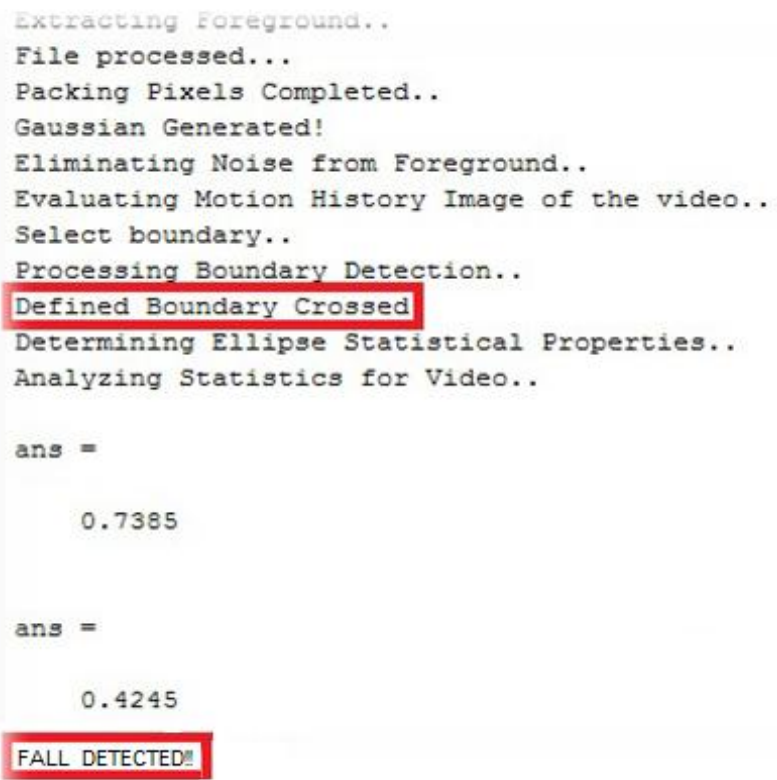

Figure. 10 Final Results

The first line enclosed by a rectangle in red color depicts the information of the child crossed the specified boundary. The second line enclosed by a rectangle in red color shows the fall detection of the child as shown below in fig 10 .

\section{ANALYSIS OF FALL DETECTION ALGORITHM}

Nicholas, Patel, Abhishek developed the fall detection method [12]. They named the proposed system "FALL DETECTION". They have limited work and limitations in the means of implementations.

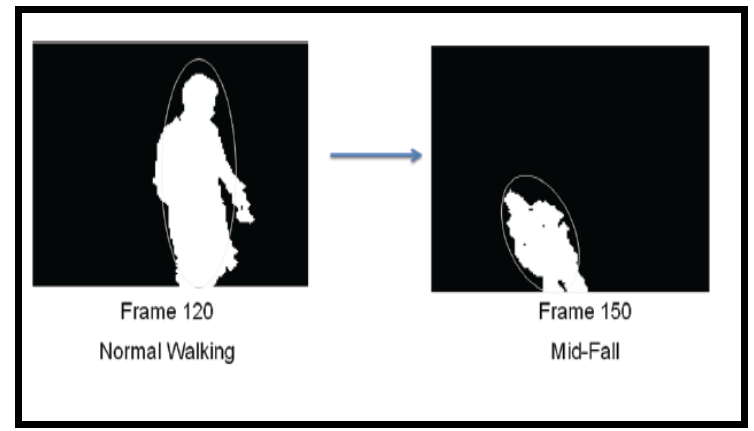

Figure 11. Man walking detection and fall detection [12]

Fig. 11 shows the normal walking of man along with the falling posture of the walking man. Fig. 12 shows the turning of man along with the falling detection.

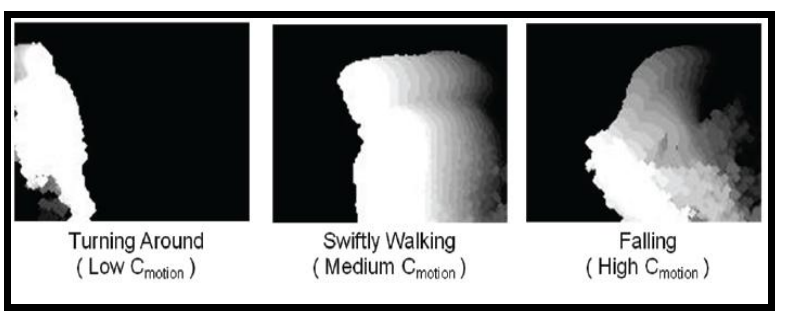

Figure 12. Man walking detection and fall detection [12] 


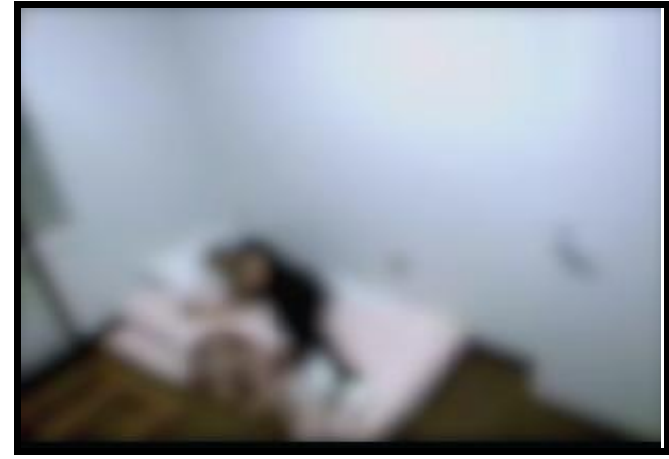

Figure. 13 Detection of Man on bed [12]
The video example from the Dataset used by them has been shown below in the fig. 13 .

The developed system [12] was too much limited in a number of cases. When the developed system was compared with the proposed system, proposed system met maximum conditions along with the success ration of fall detection. All the factors discussed in the table given below.

TABLE .1 COMPARISON OF PROPOSED ALGORITHM AND FALL DETECTION ALGORITHM

\begin{tabular}{|c|c|c|c|c|c|c|c|c|c|c|}
\hline Ser & System & $\begin{array}{c}\text { No of } \\
\text { Videos }\end{array}$ & $\begin{array}{c}\text { Fall } \\
\text { Detection }\end{array}$ & $\begin{array}{c}\text { Non Fall } \\
\text { Detection }\end{array}$ & $\begin{array}{c}\text { Processing } \\
\text { Time }\end{array}$ & Lights & $\begin{array}{c}\text { Without } \\
\text { Light }\end{array}$ & Cost & $\begin{array}{c}\text { Success } \\
\text { Ratio }\end{array}$ & $\begin{array}{c}\text { Frontal/Back } \\
\text { View }\end{array}$ \\
\hline 1 & $\begin{array}{c}\text { Fall } \\
\text { Detection }\end{array}$ & 50 & $\begin{array}{c}\text { Walking } \\
\text { Man only }\end{array}$ & $\begin{array}{c}\text { Walking } \\
\text { Man only }\end{array}$ & $27 \mathrm{sec}$ & Must & No & $1000 \$$ & $83.3 \%$ & Frontal only \\
\hline 2 & Proposed & 200 & $\begin{array}{c}\text { Child Fall } \\
\text { Detection }\end{array}$ & Child & $15 \mathrm{sec}$ & Yes & Yes & $270 \$$ & $89.4 \%$ & Both \\
\hline
\end{tabular}

The "Fall Detection" system was limited to walking man and under light conditions with must HD camera. The Processing time was much slower than the developed system. Fall detection success ratio was not of much difference with the proposed system but proposed system has not too much limitation as compared to the "Fall Detection System". Fig. 14 depicts the success ratio between our proposed system and "Fall Detection Method" of Nicole and Abhishek[12].

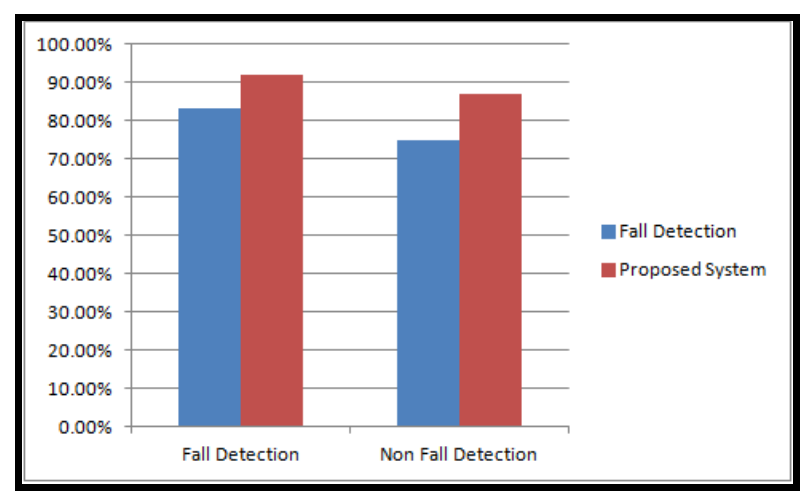

Figure. 14 Success Ratio

Fig. 14 describes the success ratio of both systems whereas Fig. 15 describes the failure ration. Both clearly speaks that Proposed System (Child Fall Detection) is far better than "Fall Detection Method"[12].

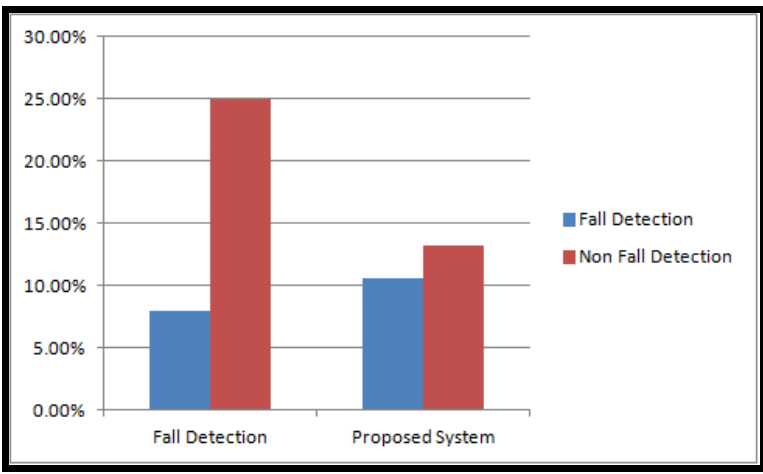

Figure. 15 Failure Ratios

\section{CONCLUSION}

For efficient detection of Child fall in the room and absence recognition of child we have designed the system which detects the Child in the first phase, continuously monitors the movements of child and generates an Alarm message in case of Child Fall Detection. The proposed system also considers the absence recognition of child from a specified portion selected in the room. The proposed system achieves the task of fall detection and absence recognition in an efficient and effective way which has been proved by the results. It also provides the solution for detecting the falling of patients and handicap in hospitals. It can also be used for academic and scientific research.

\section{REFERENCES}

[1] Zhuolin Jiang, Shaofa Li, Dongfa Gao. A Time Saving Method for Human Detection in Wide Angle Camera Images. International Conference on Machine Learning and Cyber-netics, 2006. 
[2] N. Dalal and B. Triggs. "Histograms of oriented gradients for human detection," Conference on Computer Vision and Pattern Recognition (CVPR) 2005.

[3] Paul Viola, M Jones, ' Rapid object detection using a boosted cascade of simple features", published in Computer Vision and pattern recognition, 2001.

[4] Chong Chen, Schonfeld, D., "A Particle Filtering Framework for Joint Video Tracking and Pose Estimation Image Processing", IEEE Transactions, pp. 1625 - 1634, June 2010.

[5] Muhammad Jamil Khan and Hafiz Adnan Habib, "Video Analytic for Fall Detection from Shape Features and Motion Gradients", Proceedings of the World Congress on Engineering and Computer Science 2009 Vol II WCECS 2009, October 20-22, 2009, San Francisco, USA.

[6] Mohamad Adnan Al-Alaoui, Lina Al-Kanj, Jimmy Azar, Elias Yaacoub, "Speech Recognition using Artificial Neural Networks and Hidden Markov Models", IEEE Technology and Engineering Education (ITEE), Vol 3, No 3 (2008)

[7] Li Cheng; Minglun Gong; Schuurmans, D, 'Caelli, T.; Real-Time Discriminative Background Subtraction Image Processing', IEEE Transactions, pp. 1401 - 1414, May 2011.

[8] Inter frame Coding, http://en.wikipedia.org/wiki/Inter_frame

[9] G.-X. Yuan, C.-H. Ho, and C.-J. Lin. An Improved GLMNET for L1-regularized Logistic Regression and Support Vector Machines. (supplementary materials, code). Journal of Machine Learning Research, 13(2012), 1999-2030. A short version appears at ACM KDD 2011.

[10] Bovolo, F., Bruzzone, L., Carlin, L, "A Novel Technique for Subpixel Image Classification Based on Support Vector Machine Image Processing', IEEE Transactions, pp. 2983 - 2999, Nov. 2010.

[11] David G. Lowe,'Object Recognition from Local Scale-Invariant Features" Proc. of the International Conference on Computer Vision, Corfu (Sept. 1999)

[12] Nicholas, Patel, Abhishek, Fall Detection system, "http://www.ece.gatech.edu/academic/courses/ece4 007/09spring/ece4007105/ak9/"

Engr. Ali Javed is serving as an Assistant professor in the Department of Software Engineering at University of Engineering \& Technology, Taxila, Pakistan. He is also a PhD Scholar in Computer Engineering Department at University of Engineering \& Technology, Taxila, Pakistan. He has received his MS degree in Computer Engineering from University of Engineering \& Technology, Taxila, Pakistan in February, 2010. He received Chancellor's Gold Medal in MS Computer
Engineering degree and became the first MS student in the history of UET Taxila to be awarded Chancellor's Gold Medal. He has received B.Sc. degree in Software Engineering from University of Engineering \& Technology, Taxila, Pakistan in September, 2007. He got 3rd position in Software Batch-2003F in BS Software Engineering degree. His areas of interest are Digital Image Processing, Computer vision, Video Summarization, Software Requirements Analysis, Software Design, Software Quality Assurance and Software testing.

Engr. Rabeea Islam is MS Student in the Department of Software Engineering, University of Engineering and Technology Taxila, Pakistan. She graduated from University of Engineering and Technology Taxila in Software Engineering in September 2007. Her areas of interest are Digital Image Processing and Computer vision. 